\section{Cahiers de Narratologie}

Analyse et théorie narratives

$30 \mid 2016$

Street Art 2

\title{
Compte rendu de lecture de l'ouvrage de Clément, Bruno, Härle, Clemens-Carl (dirs), Aux confins du récit
}

\section{Marc Marti}

\section{OpenEdition}

\section{Journals}

Electronic version

URL: http://journals.openedition.org/narratologie/7528

DOI: $10.4000 /$ narratologie. 7528

ISSN: 1765-307X

Publisher

LIRCES

\section{Electronic reference}

Marc Marti, « Compte rendu de lecture de l'ouvrage de Clément, Bruno, Härle, Clemens-Carl (dirs), Aux confins du récit », Cahiers de Narratologie [Online], 30 | 2016, Online since 28 July 2016, connection on 02 May 2019. URL : http://journals.openedition.org/narratologie/7528; DOI : 10.4000/ narratologie. 7528

This text was automatically generated on 2 May 2019.

Article L.111-1 du Code de la propriété intellectuelle. 


\title{
Compte rendu de lecture de l'ouvrage de Clément, Bruno, Härle, Clemens-Carl (dirs), Aux confins du récit
}

\author{
Marc Marti
}

\section{REFERENCES}

Clément, Bruno, Härle, Clemens-Carl (dirs), Aux confins du récit, Paris, Presses universitaires de Vincennes, 2014

1 Les articles qui composent cet ouvrage collectif sont des textes issus des communications présentées en 2008, lors du colloque Limiti del raconto, organisé à l'université de Sienne, en partenariat avec le collège international de philosophie de Paris. Une première version des actes a été publiée en italien sous le titre Confini del raconto (Macerata, Quodlibet, 2009).

2 Comme l'indique le titre du livre, il s'agit de cerner un peu mieux l'indéfinition de la notion de récit en explorant ses limites. La première originalité consiste à avoir mobilisé non seulement des spécialistes de la littérature, mais aussi des chercheurs en arts et en philosophie, une façon d'établir et en même temps de réfuter ce qui serait une marge disciplinaire dans les études sur le récit. Cette pluridisciplinarité très stimulante donne lieu à trois types de contributions.

3 Un premier groupe de travaux propose plutôt des mises au point à travers de larges synthèses, qui font appel aux apports du dernier demi-siècle en matière de ce que l'on peut appeler la narratologie, bien que le terme soit plutôt issu des forges littéraires et linguistiques. Guido Mazoni et Alexander García Düttman pour la philosophie, Tala Pecker Berio pour la musicologie et Clemens Carl Härle pour la littérature reviennent sur les textes fondateurs de leur discipline sur la question. Bruno Clément (philosophie) 
quant à lui interroge les fondements même des disciplines en postulant que « toutes les disciplines de l'esprit, ou du sens, seraient de même filles du récit, enfants de la narration », ce qui l'amène à forger un "mythe inexistant [qui] parlerait d'abord d'une époque lointaine et somme toute heureuse, une sorte d'âge d'or, où la pensée n'était pas distincte du récit, ni le récit de la pensée ».

D'autres études proposent d'explorer de nouvelles notions ou de nouveaux supports. C'est le cas de l'article de Jean-Luc Nancy sur le récitatif ou de ceux d'Omar Calabrese et de Patrick Vaudray, qui reviennent sur les rapports entre littérature et peinture. Le premier le fait en étudiant la narrativité picturale en relation avec des procédés et des codes historique, tandis que le second propose d'inverser le Ut pictura poesis à travers l'analyse de l'œuvre de Manet.

5 La dernière série de travaux est constituée par des recherches monographiques, toujours sur les confins du récit, identifiés de différentes façons. Il s'agit de la question du genre littéraire pour Catherine Maubon, qui analyse l'autobiographie chez Leiris ainsi que pour Isabelle de Fontenay qui pointe l'oscillation entre fiction et non fiction dans Les disparus de David Mendelsohn. Pierre Drogi s'intéresse au traitement du temps et à son rapport avec le personnage chez Dostoïevski. Enfin Françoise Asso, dans une démarche comparative, fait émerger la limite entre le renoncement au récit et l'impossibilité de récit chez certains auteurs du XIXe et du XXe siècle. 